This item was submitted to Loughborough's Research Repository by the author.

Items in Figshare are protected by copyright, with all rights reserved, unless otherwise indicated.

\title{
Radiation efficiency of natural modes of plates with beam stiffeners
}

PLEASE CITE THE PUBLISHED VERSION

https://www.sfa.asso.fr/fr/accueil

\section{PUBLISHER}

Société Française d'Acoustique / The French Society of Acoustics

\section{VERSION}

AM (Accepted Manuscript)

\section{LICENCE}

CC BY-NC-ND 4.0

\section{REPOSITORY RECORD}

Rousounelos, Andreas, Stephen J. Walsh, and Victor V. Krylov. 2011. "Radiation Efficiency of Natural Modes of Plates with Beam Stiffeners". figshare. https://hdl.handle.net/2134/8165. 

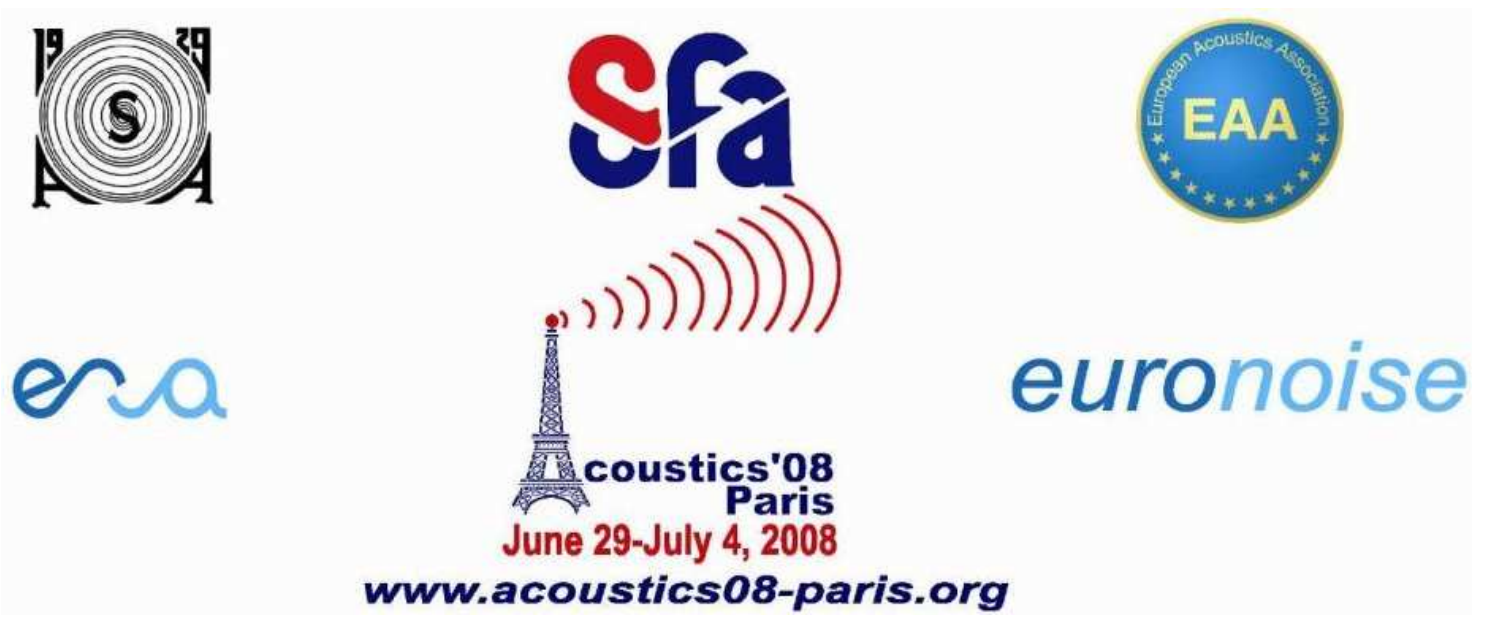

\title{
Radiation efficiency of natural modes of plates with beam stiffeners
}

\author{
A. Rousounelos, S. J Walsh and V. V. Krylov
}

Loughborough University, Department of Aeronautical and Automotive Engineering, Ashby Road, LE11 3TU Loughborough, UK

a.rousounelos@lboro.ac.uk 
In this paper, the radiation efficiency of the natural modes of finite plates with attached beams is considered. Firstly, the scattering of flexural waves, by the beams and the boundaries, in the plate is considered. The reflection and transmission coefficients along with the near-field coefficients of the beam are used to calculate the vibration field of the plate, by taking into account an infinite number of reflections from the boundaries. A wavenumber transform of the vibration velocity field is used to calculate the sound power and the radiation efficiency. The stiffening beams will shift the resonant frequencies of the plate to higher or lower frequencies depending upon the resonant frequency of the beam. This will also affect the radiation efficiency. From numerical evaluation, it is shown that the beam behaves as an added stiffness for frequencies below the resonant frequency of the beam stiffener whereas for frequencies above this frequency the beam behaves as an added mass.

\section{Introduction}

Beam-stiffening is a common technique used as an effort to minimise the acoustic radiation of plates, by modifying its structural properties. Although beam-stiffening has been widely used in many applications for sound and vibration control, its effects on sound radiation has not been fully studied theoretically. Therefore, sometimes the use of beam-stiffening might lead to undesired acoustic results.

One of the first and most important publications regarding sound radiation by beam-stiffened plates is that by Maidanik [1]. By studying the acceleration spectrum, and its relation to the sound power, he showed that the discontinuities of the plate at the beam's location will, in general, increase the coupling between the structure and the surrounding acoustic medium. Maidanik also derived approximate expressions for the radiation efficiency of the beam-plate system as a function of frequency, by employing a statistical method, which led to the development of a then new technique later called Statistical Energy Analysis.

The interaction of flexural waves, in an infinite panel, with a beam discontinuity was studied by Ungar [2]. Expressions for the transmission, reflection and near-field effects are presented and are related to trace matching between flexural waves in the plate with flexural and torsional waves in the beam. Lyon [3] used these expressions, to investigate the radiation resistance of the infinite beamplate system, for different configurations of the beam.

Although the study of infinite beam-plate systems, as presented in [2],[3], reveals the effects of an attached beam to the vibration and acoustic radiation of a plate, it does not take into account the modal behaviour of the beam. This is considered by Heckl [4], where a plate infinite in one dimension and finite in the other dimension, with attached beams across the finite dimension is studied. Expressions for the transmission and reflection coefficients are presented, in order to discuss the wave propagation along the complex system. The near-field effects are not taken into account as they are local and do not propagate away from the discontinuity. However, these effects must be considered for the study of sound radiation of the structure, as the near-field is able to radiate sound.

A number of publications are concerned with the sound radiation by periodically stiffened infinite plates [6],[7],[8],[9]. Analytical solutions for the vibration and the radiated sound pressure in the far-field is given for the case where the structure is excited by harmonic pressure [6] and by point or line forces [7],[8],[9],[10]. However, in many practical situations, such as panels in automotive structures, the excitation is much more complicated than simple point or line forces. Hence, a more general approach is required.

More recently, some researchers [11],[12],[13] have focused on the numerical optimisation of the acoustic design of a structure, by taking advantage of the increasing capabilities of microprocessors. The proposed methods use optimisation algorithms, such as genetic algorithms, and numerical methods such as the finite element method and/or the boundary element method, to optimise the position and properties of a beam attached onto a plate. Although, the acoustic optimisation techniques are very powerful for dealing with problems where the parameters (such as, source excitation, geometry of the structure, etc) are specific and they are known a priori, they are unable to shed light on the physical phenomena of sound radiation by beam-stiffened plates and hence to give general guidelines for designing such structures.

In this paper, the vibration field of a finite simply supported plate with attached stiffeners is considered by assuming a propagating wave in the plate, in the direction perpendicular to the beam, and the scattering caused by the beams along with an infinite number of reflections from the boundaries. In the direction of the beam, the vibration field is considered to be one of the mode shapes of the simply supported plate. In this way, the structure is assumed to be excited with an equal amount of energy at all frequencies, hence all cases of specific excitation, such as point, line or sound excitation, can be considered as sub-cases of this general approach. Once the velocity spectrum of the structure is known, its radiation efficiency can be calculated. Figures of the radiation efficiency with respect to frequency for different mode numbers in the direction of the beam are presented.

\section{Wave propagation along the beam- plate system}

Wave propagation has been studied by Ungar [2] for an infinite beam-plate system and by Heckl [4] and Rousounelos, et al [14], for a structure finite in the dimension along the beam and infinite in the other dimension. A similar procedure to the latter is adopted here for a finite stiffened plate in all dimensions. A flexural wave is assumed to exist in the plate travelling in the positive $\mathrm{x}$-direction. If the plate has no boundaries in the $\mathrm{x}$ dimension then the vibration field of the structure consists of the travelling wave plus the reflected, transmitted and evanescence waves due to the beam discontinuity. In the ydirection, the direction where the beam lies, the displacement is given by the summation of an infinite number of modes. For the present study the boundaries are 
assumed to be simply supported hence the displacement is given as an infinite series of sinusoidal functions. For a beam lying at $\mathrm{x}=0$, using standard mathematical notation the displacement along the beam-plate system is:

$w(x, y)= \begin{cases}\sum_{n} a_{n} \sin \frac{n \pi y}{L_{y}}\left[\begin{array}{ll}\left.\exp \left(-i\left(k_{n}\left(x-x_{b 1}\right)+\pi / 2\right)\right)+R_{n} \exp \left(i\left(k_{n}\left(x-x_{b 1}\right)-\pi / 2\right)\right)\right) \\ +R_{n}^{\prime} \exp \left(k_{n}^{\prime} x\right)\end{array}\right. & x<0 \\ \sum_{n} a_{n} \sin \frac{n \pi y}{L_{y}}\left[T_{n} \exp \left(-i\left(k_{n}\left(x-x_{b 2}\right)+\pi / 2\right)\right)+T_{n}^{\prime} \exp \left(-k_{n}^{\prime} x\right)\right] & x>0\end{cases}$

with $k_{n}^{2}=k_{p}^{2}-\left(\frac{n \pi}{L_{y}}\right)^{2}$ and $k_{n}^{\prime 2}=k_{p}^{2}+\left(\frac{n \pi}{L_{y}}\right)^{2}$

The coefficients $R_{n}$ and $T_{n}$ are the complex reflection and transmission coefficients respectively and $R_{n}^{\prime}$ and $T_{n}^{\prime}$ are the corresponding near-field coefficients. The subscript $n$ indicates that the coefficients depend on the mode-number in the y-dimension. The expansion coefficient $a_{n}$ depends on the excitation of the plate. The rest of the variables will be explained later in this section.
Forces and moments exerted on the beam by the plate's vibration have been described by Ungar [2]. Equating these forces and moments of the beam with that of the plate for both sides of the structure (positive and negative $x$ ), results in four equations which can be solved simultaneously to give the four unknown coefficients $R_{n}, T_{n}, R_{n}^{\prime}$ and $T_{n}^{\prime}$. Thus,

$$
\begin{aligned}
& R_{n}=\frac{\exp \left(-i k_{n} L_{x}\right)\left(k_{n}-i k_{n}^{\prime}\right)\left(\text { tors } \cdot \text { flex }+2 D k^{\prime} L_{y}^{2}\left(\text { flex }+k_{n}^{2} L_{y}^{2} \text { tors }\right)\right)}{\left(k_{n}+i k_{n}^{\prime}\right)\left(2 D\left(k_{n}^{\prime}+i k_{n}\right) L_{y}^{2}+\text { tors }\right)\left(2 D k_{n}\left(k_{n}-i k_{n}^{\prime}\right) k_{n}^{\prime} L_{y}^{4}+\text { flex }\right)} \\
& T_{n}=\frac{\exp \left(-i k_{n} L_{x}\right) 2 i D k_{n}\left(k_{n}-i k_{n}^{\prime}\right) L_{y}^{2}\left(2 D k_{n}^{\prime}\left(k_{n}^{2}+k_{n}^{\prime 2}\right) L_{y}^{4}+\text { flex }+k_{n}^{\prime 2} L_{y}^{2} \text { tors }\right)}{\left(k_{n}+i k_{n}^{\prime}\right)\left(2 D\left(k_{n}^{\prime}+i k_{n}\right) L_{y}^{2}+\text { tors }\right)\left(2 D k_{n}\left(k_{n}-i k_{n}^{\prime}\right) k_{n}^{\prime} L_{y}^{4}+\text { flex }\right)} \\
& R_{n}^{\prime}=\frac{2 i k_{n}\left(D\left(k_{n}-i k_{n}^{\prime}\right) L_{y}^{2}\left(i f l e x+k_{n} k_{n}^{\prime} L_{y}^{2} \text { tors }\right)+\text { tors } \cdot \text { flex }\right)}{\left(k_{n}+i k_{n}^{\prime}\right)\left(2 D\left(k_{n}^{\prime}+i k_{n}\right) L_{y}^{2}+\text { tors }\right)\left(2 D k_{n}\left(k_{n}-i k_{n}^{\prime}\right) k_{n}^{\prime} L_{y}^{4}+\text { flex }\right)} \\
& T_{n}^{\prime}=-\frac{2 D k_{n}\left(k_{n}-i k_{n}^{\prime}\right) L_{y}^{2}\left(\text { flex }+i k_{n} k_{n}^{\prime} L_{y}^{2} \text { tors }\right)}{\left(k_{n}-i k_{n}^{\prime}\right)\left(2 D\left(k_{n}^{\prime}+i k_{n}\right) L_{y}^{2}+\text { tors }\right)\left(2 D k_{n}\left(k_{n}-i k_{n}^{\prime}\right) k_{n}^{\prime} L_{y}^{4}+\text { flex }\right)}
\end{aligned}
$$

where

$$
\begin{aligned}
& \text { tors }=G K L_{y}^{2}\left(\frac{J \omega^{2}}{G K}+\left(\frac{n \pi}{L_{y}}\right)^{2}\right) \\
& \text { flex }=B L_{y}^{4}\left(\frac{m_{b} \omega^{2}}{B}-\left(\frac{n \pi}{L_{y}}\right)^{4}\right)
\end{aligned}
$$

$L_{x}$ and $L_{y}$ are the dimensions of the plate and $D$ is its flexural rigidity, $B$ is the flexural rigidity of the beam, $m_{b}$ is its mass per unit length, $G K$ is the beam torsional rigidity and $J$ is its polar mass moment of inertia. tors and

$$
w(x)= \begin{cases}\left(1-2 v_{1}\right) \sin \left(k_{n}\left(x-x_{b 1}\right)\right)-i \cos \left(k_{n}\left(x-x_{b 1}\right)\right) & -L \leq x<0 \\ +\left(v_{1} R_{n}^{\prime}-v_{2} T_{n}^{\prime}\right) \exp \left(k_{n}^{\prime} x\right) & 0<x \leq L \\ 2 v_{2} \sin \left(k_{n}\left(x-x_{b 2}\right)\right)+\left(v_{1} T_{n}^{\prime}-v_{2} R_{n}^{\prime}\right) \exp \left(-k_{n}^{\prime} x\right) & \end{cases}
$$


where

$$
\begin{aligned}
& v_{1}=\frac{1+R_{n}}{\left(1+R_{n}\right)^{2}-T_{n}^{2}} \\
& v_{2}=-\frac{T_{n}}{T_{n}^{2}-\left(1+R_{n}\right)^{2}}
\end{aligned}
$$

and $L$ is the length of each half section of the beam-plate $\operatorname{system}\left(L=L_{x} / 2\right)$.

\section{Sound Radiation}

The acoustic power, $P_{n}$, radiated by a given mode $\mathrm{n}$ of a baffled planar source can be calculated from the following expression:

$$
P_{n}=\frac{\rho c k}{8 \pi^{2}} \int_{-k-k}^{k} \int_{\sqrt{k^{2}-k_{x}^{2}-k_{y}^{2}}}^{k} d k_{x} d k_{y}
$$

where, $\rho$ is the volume density of the acoustic medium, $c$ is the speed of sound, $k$ is the acoustic wavenumber and $\left|u\left(k_{x}, k_{y}\right)\right|^{2}$ is the velocity power spectrum of the plate, given by the double Fourier transform of the spatial velocity distribution of the plate:

$$
u_{n}\left(k_{x}, k_{y}\right)=\int_{-\infty}^{\infty} \int_{-\infty}^{\infty} u_{n}(x, y) \exp \left(-i k_{x} x\right) \exp \left(-i k_{y} x\right) d x d y
$$

The radiation efficiency can then be calculated by:

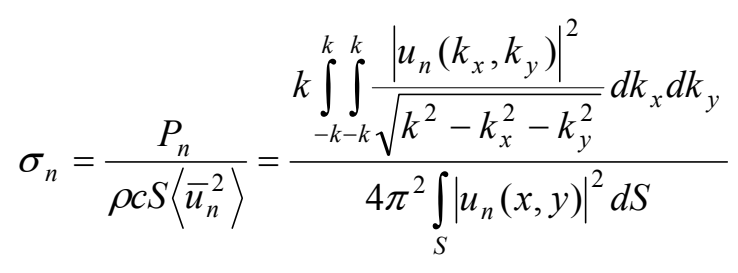

where $\left\langle\bar{u}_{n}^{2}\right\rangle$ is the mean square velocity of the structure. By multiplying Eq. (2) by $i \omega$ and taking its Fourier transform, using $\sin \frac{n \pi y}{L_{y}}$ for the y-dimension, the velocity spectrum is given by:

$$
\begin{aligned}
& u_{n}\left(k_{x}, k_{y}\right)=\frac{\left(1-(-1)^{n} \exp \left(-i k_{y} L_{y}\right)\right)}{\left(n \pi / L_{y}\right)^{2}-k_{y}^{2}} \frac{n \pi}{L_{y}} \\
& \left(\begin{array}{l}
\frac{\left(\left(k_{n}+k_{x}-2 k_{n}\left(v_{1}+v_{2}\right)\right) \cos \left(k_{n} L\right)+i\left(k_{n}+k_{x}-2 k_{x}\left(v_{1}+v_{2}\right)\right) \sin \left(k_{n} L\right)\right)-\exp \left(i k_{x} L\right)\left(k_{n}+k_{x}-2 k_{n} v_{1}\right)-\exp \left(-i k_{x} L\right) 2 k_{n} v_{2}}{k_{x}^{2}-k_{n}^{2}} \\
-\frac{\left(\exp \left(-\left(k_{n}^{\prime}+k_{x}\right) L\right)-1\right)\left(v_{1} T_{n}^{\prime}-v_{2} R_{n}^{\prime}\right)}{k_{n}^{\prime}+i k_{x}}-\frac{\left(\exp \left(-\left(k_{n}^{\prime}-k_{x}\right) L\right)-1\right)\left(v_{1} R_{n}^{\prime}-v_{2} T_{n}^{\prime}\right)}{k_{n}^{\prime}-i k_{x}}
\end{array}\right)
\end{aligned}
$$

\section{$4 \quad$ Numerical results}

Substituting $u_{n}\left(k_{x}, k_{y}\right)$ to Eq. (4), and evaluating the two integrals numerically, the radiation efficiency of the structure, for the $n^{\text {th }}$ mode in the y-direction, can be calculated. To check the validity of the model described above, the radiation efficiency calculated from Eq. (4) is compared with the radiation efficiency calculated by FEM/BEM numerical methods, using Nastran/Patran and LMS Virtual Lab software. In order to derive the radiation efficiency of the structure from the radiation efficiency of a single mode, the following expression is used [15]:

$$
\sigma=\frac{\sum_{n} \sigma_{n}\left\langle\bar{u}_{n}^{2}\right\rangle}{\sum_{n}\left\langle\bar{u}_{n}^{2}\right\rangle}
$$

In the model of the beam-plate system developed above, all modes are excited with the same amount of energy. In order for the comparison with the FEM/BEM methods to be made, the radiation efficiency of a point excited stiffened plate averaged for 10 different point force locations is calculated. The plate that is used is a steel plate with one stiffener in the middle of its length. The comparison is shown in Figure 1.

A first observation that was made by investigating the model described above is regarding the shift in the resonant frequencies of the structure caused by the beam. As it is expected, when the beam location is across the middle of the plate, the influence of the beam to the resonant frequencies of the even modes is small, as there is no displacement at the location of the beam and hence only the rotation of the beam influences these modes. For the odd modes, the beam behaves like an added stiffness, hence it shifts the resonant frequency upwards, when the resonance of the structure is lower than the resonance of the beam $\left(f_{\text {beam }}\right)$. When the resonance of the beam is lower than that of the structure, the beam behaves like an added mass, hence it shifts the resonant frequency down. Figure 2 shows the mean squared velocity of a plate, with and without a 


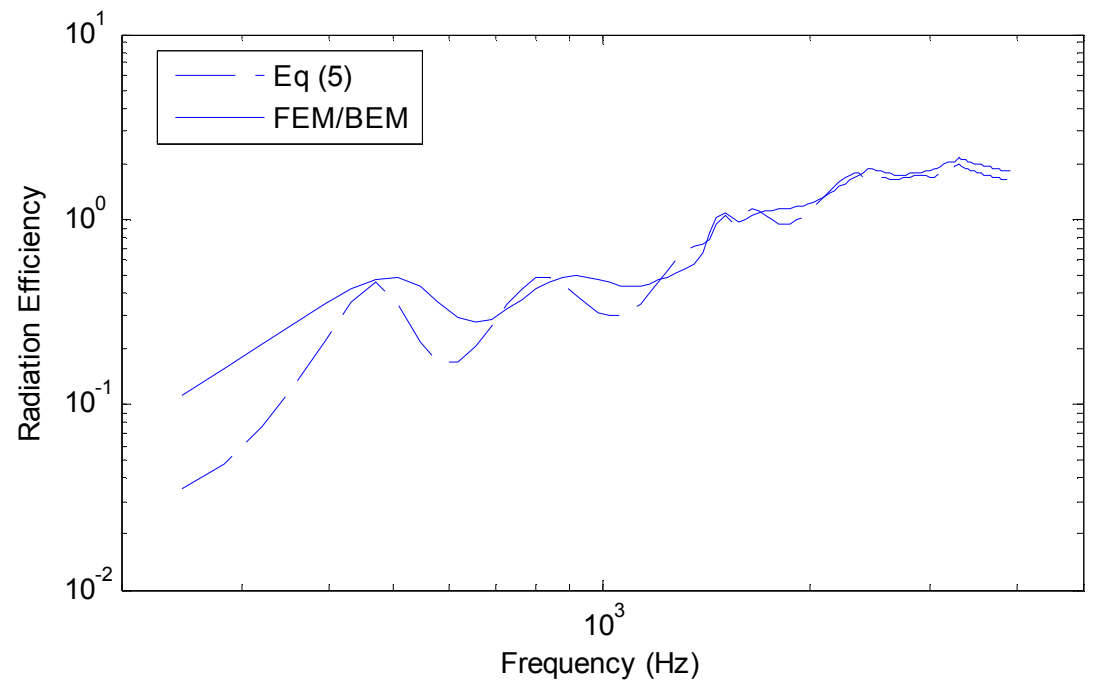

Figure 1. Comparison of radiation efficiency calculated using FEM/BEM and Eq. (4) \& (5) for a steel plate with $L_{x} \mathrm{x} L_{y}$ $307 \mathrm{~mm} \times 208 \mathrm{~mm}, 5 \mathrm{~mm}$ thick with a steel beam stiffener $15 \mathrm{~mm}$ high and $5 \mathrm{~mm}$ wide.

stiffener, for $n=1$, where the effect of the beam at resonant frequencies can be seen. The same results were obtained for other mode numbers. Note that when we refer to the resonant frequency of the beam, the resonant with mode number $n$, the mode number of the mode along the dimension of the beam, is implied.

It has been observed that the effect of added stiffness and mass is proportional to the actual stiffness and mass of the beam. For example, for a heavy beam, the shift down of the resonant frequencies above $f_{\text {beam }}$ is greater than that caused by a lighter beam. The effect that the stiffener has at the resonant frequencies is also verified by FEM calculations. Moreover, using FEM, this phenomenon has been observed for plates with more than one stiffener.

This shift in the resonant frequencies has an effect on the radiation efficiency, since it is known that, the lower the resonant frequency of a given mode shape the lower is its radiation efficiency [16]. Hence, modes that are shifted higher in frequency will have a higher radiation efficiency, and modes that are shifted lower in frequency will have a lower radiation efficiency. The difference in the radiation efficiency between an unstiffened plate and a plate with one stiffener for the modes $n=1$ is illustrated in Figure 3 . It can be also seen that for the case of one stiffener, the critical frequency remains the same (the critical frequency of the flexural waves of the plate, which cause sound radiation). The effect of the stiffener is diminished when the frequency is close to the critical frequency, where the two curves converge.

\section{Conclusion}

A model for the vibration field of a finite plate with one stiffener has been developed. The vibration field in the $\mathrm{x}-$ dimension consists of a propagating wave along with an infinite number of reflections from the boundaries as well as reflected and transmitted waves at the beam discontinuity. In the y-dimension, the direction where the beam lies, the vibration field consists of a single mode. Expressions for the reflection, transmission and near-field coefficients for the $n^{\text {th }}$ mode of the beam are also presented. The model can be also extended for the case of more than one stiffener.

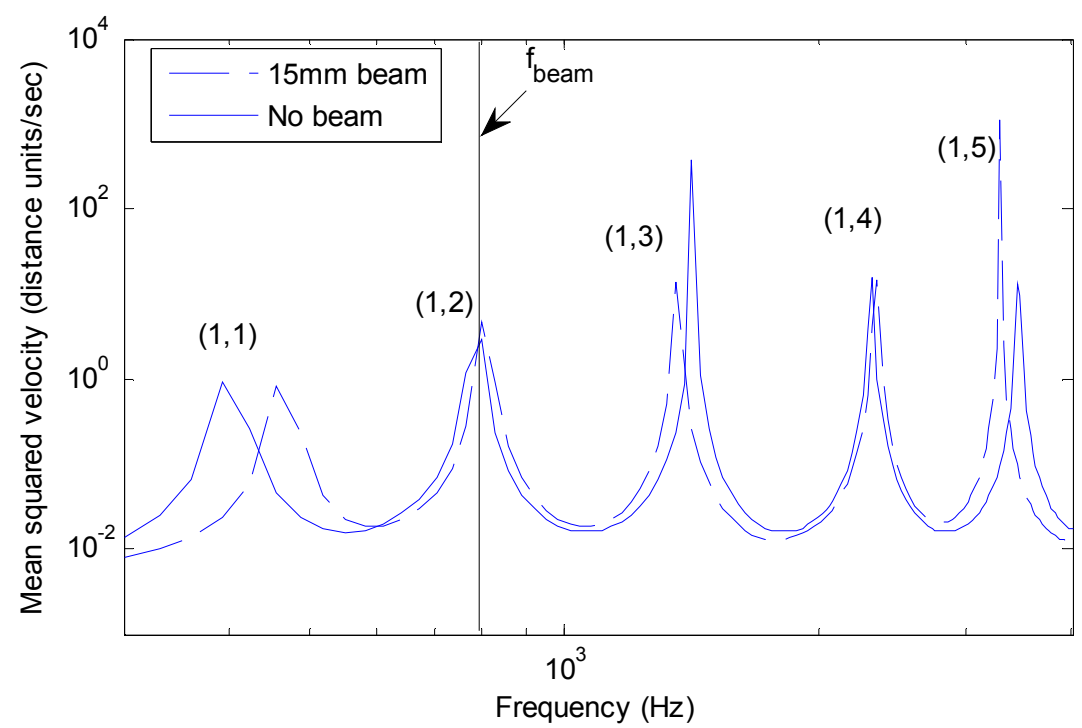

Figure 2. Mean squared velocity of a plate with the same properties as that in Figure 1 with and without the stiffener. 


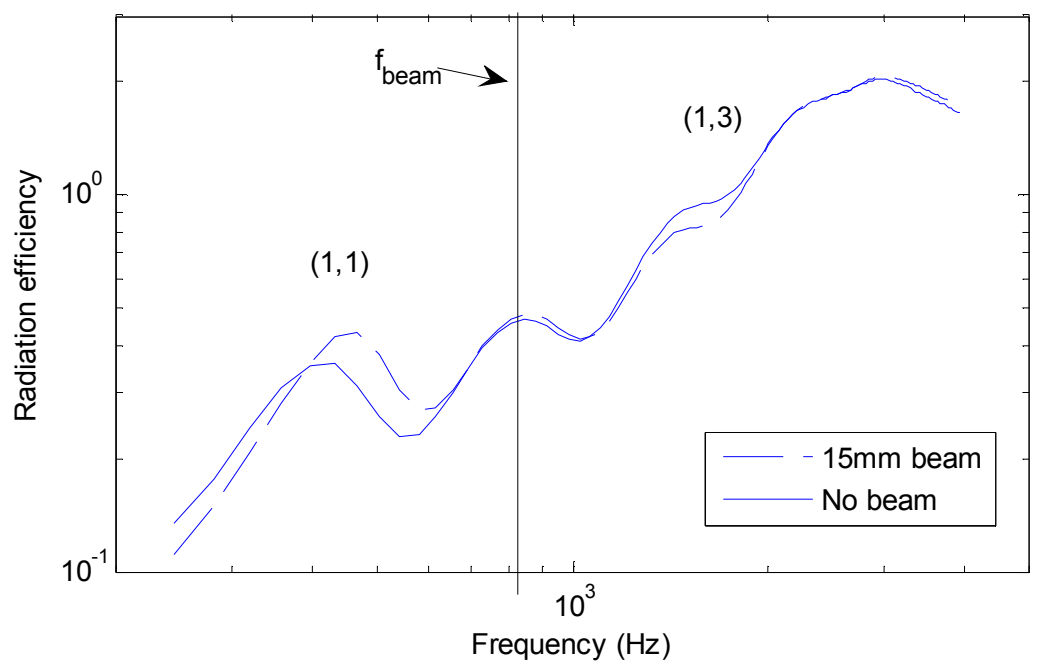

Figure 3. Radiation efficiency of a plate with the same properties as that in Figure 1with and without the stiffener for $n=1$

From the numerical evaluation of the above model, the first observation was that the resonance frequencies of the plate are shifted depending upon whether they are above or below the resonance frequency of the beam. When the resonance frequency of the plate is below the resonance frequency of the beam, the stiffener behaves as an added stiffness hence it shifts the resonant frequency higher. The opposite effect is observed when the resonance of the plate is higher than that of the beam. Then the beam behaves as an added mass hence it shifts the resonant frequency lower. The amount of added stiffness and added mass depends on the actual stiffness and mass of the beam. The shift of resonant frequencies has an effect upon the radiation efficiency as modes with lower resonance frequency have lower radiation efficiency and modes with higher resonance frequency have higher radiation efficiency. Hence, in order to reduce the radiation efficiency of a stiffened plate, the resonant frequencies of the beam must be as low as possible as well as having its mass as great as possible in order to maximise the effect. Alternative to one stiffener with great mass, more than one stiffener with low resonant frequencies can be used. Although not shown here, the results regarding the shift of the resonant frequencies have been verified using FEM for the case of more than one stiffener.

\section{Acknowledgments}

The financial support from Jaguar and Land Rover Research for this project is gratefully acknowledged.

\section{References}

[1] G. Maidanik, "Response of Ribbed Panels to Reverberant Acoustic Fields", J. Acoust. Soc. Am. 34, 809-826 (1962)

[2] E. Ungar, "Transmission of Plate Flexural Waves through Reinforcing Beams; Dynamic Stress Concentrations", J. Acoust. Soc. Am. 33, 633-639 (1961)

[3] R. Lyon, "Sound Radiation from a Beam Attached to a Plate”, J. Acoust. Soc. Am. 34, 1265-1268 (1962)
[4] M. Heckl, "Wave Propagation on Beam-Plate Systems", J. Acoust. Soc. Am. 33, 640-651 (1961)

[5] L. Cremer, M. Heckl, E.E. Ungar, Structure-Borne Sound, $2^{\text {nd }}$ Edition, Springer Verlag, Berlin, 1988, pp. 523-543

[6] B. Mace, "Periodically stiffened fluid-loaded plates, I: Response to convected harmonic pressure and free propagation", J. Sound Vib. 73, 473-486 (1980)

[7] B. Mace, "Periodically stiffened fluid-loaded plates, I: Response to line and point forces", J. Sound Vib. 73, 487-504 (1980)

[8] G. Eatwell, D. Butler, "The response of fluid-loaded, beam-stiffened plate", J. Sound Vib. 84, 371-388 (1982)

[9] V. Evseev, "Sound Radiation from an infinite plate with periodic inhomogeneities", Sov. Phys. Acoust., 19, 226-229, (1973).

[10] G. Lin, S. Hayek, “Acoustic radiation from point excited rib-reinforced plate”, J. Acoust. Soc. Am. 62, $72-83$ (1977)

[11]J. Lamancuse, "Numerical Optimization Techniques for Structural-Acoustic Design of Rectangular panels", Comput. Struct. 48, 661-675, (1993)

[12]D. Xiaolong, Z. Zongjie, Z. Xinze, "Genetic Algorithms for the Vibroacoustic Optimization of the Stamped Rib in a Plate", SAE paper 2004-01-1160

[13]D. Xiaolong, Z. Xinze, L. Li, "Vibroacoustic Optimization of Two Cross Stamped Ribs in a Plate", SAE paper 2005-01-1067

[14] A. Rousounelos, S.J. Walsh, V.V. Krylov, "Effects of beam-stiffening on the sound power radiated by finite plates", Proc. Inst of Acous 29, 42-49 (2007)

[15] G. Xie, D. Thompson, C. Jones, "The radiation efficiency of baffled plates and strips", J. Sound Vib. 280, 181-209 (2005)

[16] C. Wallace, "Radiation resistance of rectangular panel”, J. Acoust. Soc. Am. 51, 946-952 (1972) 